\title{
Cranioplasty with Methylmethacrylate in Plagiocephaly
}

\author{
So-Min Hwang, Sung-Chul Chu, Hyung-Do Kim, Min-Kyu Hwang, Min-Wook Kim, Jong-Seo Lee \\ Aesthetic, Plastic and Reconstructive Surgery Center, Good Moonhwa Hospital, Busan, Korea
}

No potential conflict of interest relevant to this article was reported.

The term plagiocephaly refers to asymmetrical distortion of a skull, or an oblique head [1]. The condition can be distinguished between congenital and acquired types. Congenital plagiocephaly results from improper timing of closure of cranial sutures, whereas asymmetric forces acting upon a child's skull (i.e., one side of the head being pressed by lying down or facing downward). In younger children with open sutures, plagiocephaly can be improved using a cranial remolding orthosis. Such treatments are not appropriate for adults, and correction of plagiocephaly requires operative intervention.

The goals of cranioplasty is to maintain the protective cage around the brain, restore the normal contour of the cranium, and improve head shape. Autologous bone and alloplastic materials are used for cranioplasty [2]. Autologous bone grafts are considered safe but requires a donor site. In addition, the surgical process is complicated, and trimming the graft to the desired shape can be difficult. Alloplastic materials include methylmethacrylate, hydroxyapatite, high-density polyethylene, titanium mesh, and solid silicone. In particular, methylmethacrylate is chemically stable, light-weight, strong, easily shapeable, and low-priced [2]. We present cranioplasty to correct plagiocephaly in an elderly patient.

A 72-year-old man presented for aesthetic correction of plagiocephaly. On examination, the cranium was asymmetric with a

Correspondence: So-Min Hwang

Aesthetic, Plastic and Reconstructive Surgery Center, Good Moonhwa Hospital, 119, Beomil-ro, Dong-gu, Busan 48735, Korea

E-mail: sominhwang@hanmail.net

Received October 11, 2015 / Revised March 2, 2016 / Accepted March 8, 2016 flat right occiput suggesting premature closure of the right lambdoidal suture, which was confirmed on the preoperative computed tomography study (Fig. 1). The patient was offered cranioplasty using methylmethacrylate, to which he readily consented.

Under general anesthesia, the scalp was opened with a bicoronal incision. The scalp flap was elevated along the subgaleal layer down to the external occipital protuberance. The portion of cranium to be augmented was designed onto the periosteum, which was opened and elevated to create the subperiosteal space for methylmethacrylate placement.

Methylmethacrylate copolymer powder and liquid monomer were mixed together and stirred to proper consistency. Approximately 50-mL implant material shaped to augment the right occiput to match the overall symmetry and placed into the subperi-
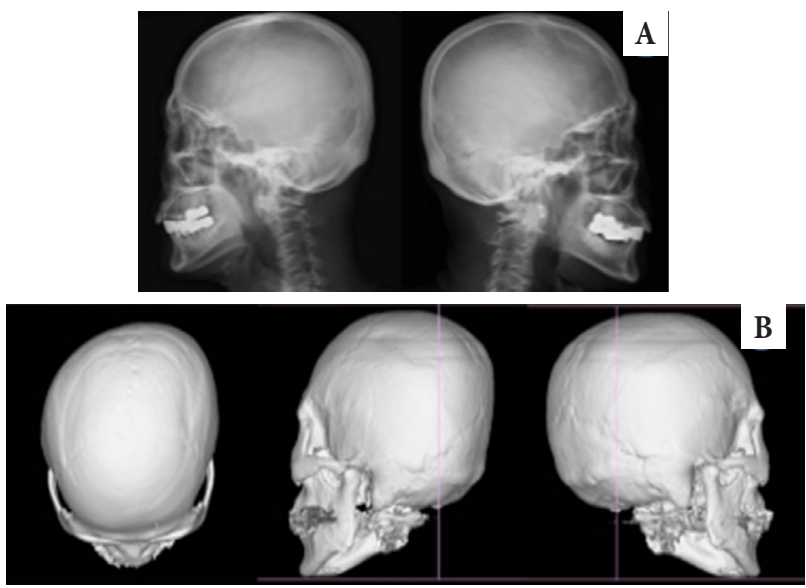

Fig. 1. (A) Preoperative X-ray findings. (B) Preoperative facial threedimensional computed tomography findings. 
osteal space.

To counteract the exothermic reaction from polymerization, the subperiosteal pocket was irrigated with cold normal saline solution. Once sufficiently cooled, the implant was fixed to the underlying cranium using two 6-mm screws, and the edges of the hardened methylmethacrylate implant was trimmed and burred. With a Hemovac drain placed superficial to the periosteum space, the periosteum and rest of the scalp were closed in successive layers.

The head was dressed with compression bandage for 2 days, and the Hemovac drain was removed on the third day. Postoperative Xray was taken on the first day (Fig. 2). At the three-month follow up, the patient was satisfied with postoperative outcomes (Fig. 3).

Whereas facial asymmetry is immediately recognizable and difficult to hide, asymmetry of the cranium can be made inconspicuous with voluminous hairstyle. Despite this, patients with plagiocephaly may still experience significant psychological stress and social impairment from extreme self-consciousness of this hidden asymmetry. In such cases, aesthetic correction of the asymmetry can be beneficial, as it was for our patient.

Methylmethacrylate was developed in 1939 and was first used by Kleinschmidt in 1940 [3]. The material is used worldwide, mainly in cranial reconstruction. When the powder and liquid are mixed, the polymer takes time to become hardened and can be molded during this time inside and outside of the body. One advantage of the methylmethacrylate to metallic implants is that the polymer-based implant does not interfere with radiologic examination.

Variety of materials are reported for use in cranioplasty $[2,4]$. Autologous bone graft has the advantages inherent to the use of living tissues, including decreased risk for infection and the possi-

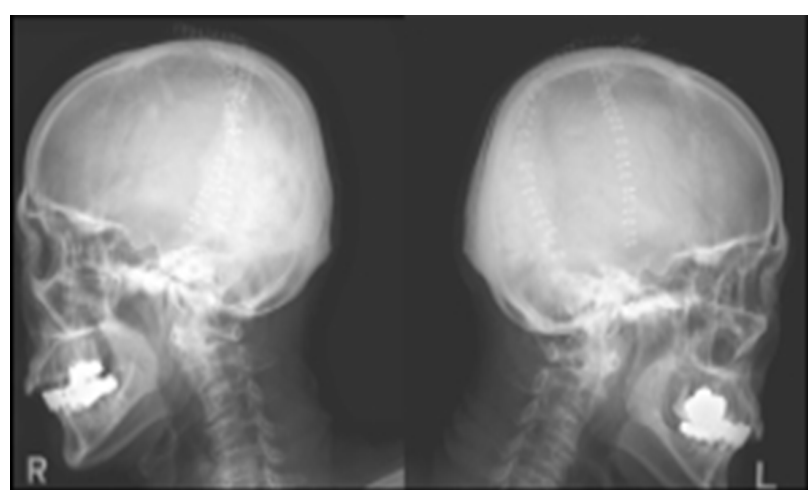

Fig. 2. Postoperative $\mathrm{X}$-ray findings.
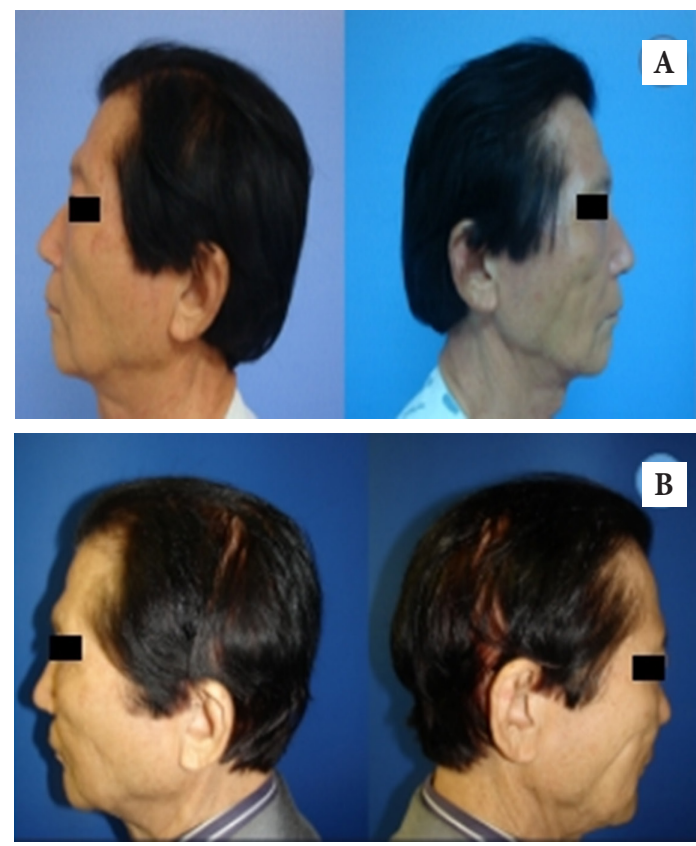

Fig. 3. (A) Preoperative clinical photo. (B) Postoperative 3 months clinical photo.

bility of organic growth in pediatric patients [4]. On the other hand, it requires a donor site bony defect without exception, and postoperative resorption can be unpredictable.

Silicone implant is used in accordance with individual patients. However, because trimming silicone implant to the exact head shape is difficult, dead space develops between the silicone implant and the skull after insertion of the implant. In addition, the edge of a silicone implant may be palpable if the soft tissue envelope is relatively thin.

The advantage of hydroxyapatite is that its structure and chemical composition are similar to those of natural bone, with excellent osteoconductive properties. In addition, the material is relatively inexpensive and easy to store. Most of all, it is easy to apply and facilitates rapid ossification. However, its use is difficult limited for cranioplasty due to lack of elasticity and size limitations.

Heat is generated when methylmethacrylate is hardened by polymerization. To minimize the risk of damage to the surrounding tissue, the heat should be removed with adequate irrigation using normal saline solution. Otherwise, exothermic reaction is proportionate to the amount of methylmethacrylate, thus im- 
plants molded within the body should not be made thicker than

$5-6 \mathrm{~mm}$ at any point [5].

The authors believe that cranioplasty using methylmethacrylate is a simple and effective method to correct depressed skull deformities.

\section{REFERENCES}

1. Teichgraeber JF, Ault JK, Baumgartner J, Waller A, Messersmith M, Gateno J, et al. Deformational posterior plagiocephaly: diagnosis and treatment. Cleft Palate Craniofac J 2002;39:582-6.

2. Kang SJ, Park HJ, Kang CS, Kim SK. Case report of cranioplasty by composite use of alloplastic materials. J Korean Cleft Palate-Craniofac Assoc 2002;3:197-200.

3. Shah AM, Jung H, Skirboll S. Materials used in cranioplasty: a history and analysis. Neurosurg Focus 2014;36:E19.

4. Han SE, Lim SY, Pyon JK, Mun GH, Bang SI, Oh KS. Aesthetic refinement of secondary cranioplasty using methyl methacrylate bone cements. Aesthetic Plast Surg 2013;37:592-600.

5. Song YT. Using methyl methacrylate in forehead contouring for aesthetic purposes. J Craniofac Surg 2013;24:1622-7. 